\title{
Les Mythologies au miroir du temps futur
}

\author{
Irène Langlet
}

\section{OpenEdition}

Journals

Édition électronique

URL : http://journals.openedition.org/recherchestravaux/432

DOI : 10.4000/recherchestravaux.432

ISSN : 1969-6434

\section{Éditeur}

UGA Éditions/Université Grenoble Alpes

\section{Édition imprimée}

Date de publication : 20 décembre 2010

Pagination : 105-117

ISBN : 978-2-84310-187-8

ISSN : 0151-1874

\section{Référence électronique}

Irène Langlet, «Les Mythologies au miroir du temps futur », Recherches \& Travaux [En ligne], 77 | 2010 mis en ligne le 20 août 2012, consulté le 08 septembre 2020. URL : http://journals.openedition.org/ recherchestravaux/432 ; DOI : https://doi.org/10.4000/recherchestravaux.432 
Irène LANGLET

Université Rennes 2

\section{Les Mythologies au miroir du temps futur}

Faut-il nécessairement être fâché avec le réel pour critiquer ses «mythologies»? Entendues à la manière barthésienne, elles désignent toutes les concrétions matérielles (objets et images) et immatérielles (comportements et pensées) où l'idéologie petite-bourgeoise adopte l'apparente évidence du naturel, de «cequi-va-de-soi ", qui a toujours-déjà eu cours, et dont le sens, pour reprendre l'une des images les plus saisissantes du recueil de 1957, est figé comme de la sauce sur un rôti photographié dans le magazine Elle. Saisissante à plus d'un titre, l'image rappelle notamment ce que les deux avant-propos de 1957 et de I970, ainsi que le texte final du recueil (mais aussi certains biographèmes de Roland Barthes par Roland Barthes'), suggèrent chacun à leur manière : que le mythologue est immergé dans la réalité qu'il s'évertue à démystifier, et qu'il n’est pas indemne du délice naturalisant que le "mythe» lui tend. Appétissants comme la sauce du rôti, le bifteck et les frites, le Tour de France ou le visage de Garbo ne révèlent leur figement qu'après l'opération d'écriture que l'essai barthésien encode alors à merveille : l'écriture personnelle et/ou imagée rend compte d'une proximité, d'un goût, d'une adhésion complète, jouissances fusionnelles avec ce réel si appétissant, juste assez longtemps pour permettre à la clausule de désigner le nœud idéologique (le gras figé sur la sauce). C'est là une composante essentielle de l'essai barthésien, peut-être irrémédiablement périmée (mais ce serait un autre débat ${ }^{2}$ ) : que le temps du texte puisse

I. R. Barthes, Roland Barthes, coll. «Écrivains de toujours», Seuil, I975.

2. On rappelle à loisir l'échec des «Mythologies, deuxième série» en 1978 dans le Nouvel Observateur. Mais quid du filon éditorial jamais démenti de ce type d'opus? Citons entre autres : O. Poivre d'Arvor, Les dieux du jour : essai sur quelques mythologies contemporaines, Denoël, 1985; M.-A. Combesque et I. Warde, Mythologies américaines, Le Félin, 1996; S. Tisseron, Petites Mythologies d'aujourd'hui, Aubier-Montaigne, 2000; J. Garcin, Nouvelles Mythologies, Seuil, 2007... 
donner corps au temps de la conscience critique personnelle, au léger délai de son émergence face au spectacle d'abord heureux du «Naturel», et que l'on récupère ainsi, dans les clausules - c'est-à-dire de façon très rarement développée, plutôt à la manière d'illuminations nécessaires et suffisantes -, le temps de l'historicité manquante. Une fois réouvert par l'essai, ce temps historique laisse toute latitude à l'historien, au sociologue, au savant, de dérouler le fil des causes et des effets qui expliquent la mystification (l'expropriation coloniale sous la belle robe du vin $\left.{ }^{3}\right)$ : le mythologue essayiste, lui, a rempli sa mission. Et celle-ci passe, on ne peut l'éluder, par un temps de l'adhésion au réel et à ses mythes.

Mais suffit-il de rester un temps devant le spectacle du mythe pour voir se craqueler son "nappé»? Autrement dit, suffit-il de réinsérer les mythologies dans une histoire, fût-elle celle, a minima, de leur contemplation d'abord ravie, pour opérer leur démystification? Leur mise en roman serait alors tout indiquée : on pourrait se dire qu'au prisme du narratif, la fixité d'image qu'impose l'opération de naturalisation se flouterait, serait embarquée dans un flux événementiel, et la plausibilité du temps qui passe replacerait comme automatiquement dans l'historicité ce qui tient si fort à y échapper. Toutefois l'affaire n'est évidemment pas si simple, et tout roman n'est pas, loin s'en faut, le lieu d'une prise de conscience idéologique. Par exemple, tout ce qui «fait tableau» dans le roman peut donner un refuge idéal au mythe et à ses chatoiements. Cependant, le procès descriptif peut, et c'est bien là toute la complication, être à son tour l'occasion d'un développement textuel qui désigne ce qu'un beau décor peut avoir de profondément idéologique, en faisant mine de l'aménager en détail. C'est une lecture fréquente du roman de Perec, Les Choses. À l'inverse (rien n'est simple), les éléments "mythologiques» (non développés) des scripts et scénarios qui permettent, selon $\mathrm{Eco}^{4}$, l'intelligibilité d'un récit romanesque plus vaste, offrent des écrins providentiels pour ces concrétions idéologiques qui transforment, par exemple (pour rester un peu chez Perec), toute «sortie dans les magasins» en un étalage quasi automatique de profusion consumériste, ou de n’importe quel «sauvetage de la planète» (pour passer à un autre type romanesque) un rappel subliminal de schèmes idéologiques : hiérarchisation brutale des classes sociales, des rôles sociaux de sexe (gender) ou des cultures nationales. Les histoires de surhommes, qui occupèrent considérablement le sémioticien italien, et le genre sciencefictionnel tout entier (notamment dans sa sous-catégorie la plus rentable, le space opera), sont même apparemment le terrain d'enquête idéal, et l'exemple tout trouvé, pour qui viendrait à soupçonner la narration de n'être pas si naturellement un procès historicisant - et donc, peut-être, démystifiant. Du

3. R. Barthes, "Le vin et le lait», Mythologies, Seuil, 1957.

4. U. Eco, Lector in fabula, Grasset, 1985. 
pénétrant "Nautilus et Bateau ivre» des Mythologies, qui replace les visions progressistes d'un Jules Verne dans le texte foisonnant de leur "moment dialectique [bourgeois d']asservissement général de la Nature ", jusqu'aux analyses de Fredric Jameson, en passant par la polémique exemplairement illustrée (tant dans la forme que sur le fond "freudo-marxiste») par Boris Eizykman dans Science-fiction et capitalisme (Mame, 1973), la science-fiction a nourri de nombreuses lectures stigmatisant sa propension à fixer perversement, sous couvert d'un imaginaire futuriste, les artefacts et habitus d'une époque et d'un milieu transformés en repères inamovibles, car non explicités, des temps présents et futurs. Il y a bien en effet de quoi douter de la capacité historicisante du roman lorsque l'imaginaire scientifique en apparence le plus audacieux ne parvient guère à installer autre chose, dans son romanesque futuriste, que des hommes d'affaires partant travailler le matin (leur bureau fût-il situé dans la plus haute tour d'une planète de la galaxie d'Andromède) pendant que leur épouse arrange des tableaux dans son salon - ces derniers fussent-ils holographiques ou à $x$ dimensions. La récente parution en recueil des articles que le marxiste américain Fredric Jameson a consacré à la science-fiction permet avantageusement de prendre la mesure du problème. Offertes à des lecteurs nourris préalablement de ses écrits sur la postmodernité et le capitalisme tardif ${ }^{6}$, ces brèves études datées pour la plupart des années 1970-I980 tournent toutes autour d'un paradoxe narratif de la science-fiction et de ce qu'il met en lumière : l'irrésistible inscription de l'infrastructure idéologique du présent dans la projection futuriste, et les diverses voies que cette dernière aménage - soit pour y opposer une résistance de type paranoïde (lectures panoptiques de Philip K. Dick), soit pour s'y abandonner en pleine acceptation de la contradiction (analyses des romans sur le thème des vaisseaux-arches).

Projeter de façon romanesque dans le futur toutes les mythologies quotidiennes dans lesquelles trouve à s'incarner une idéologie peut ainsi très efficacement contribuer à leur naturalisation. C'est ce contre quoi toute l'école dite de la "nouvelle science-fiction française politique» (NSFFP) des années I975 à I980 s'est élevée, non sans polémique, dans des recueils collectifs aux titres soigneusement étudiés comme Retour à la terre (trois volumes dirigés par Jean-Pierre Andrevon, 1975-1977) ou Futurs au présent (ouvrage dirigé par Philippe Curval, 1978). D'abord accueilli par l'une des collections de référence du genre, «Présence du futur» chez Denoël, une partie de ce vivier de nouvellistes militants migre en 1977 chez l'éditeur suisse Kesselring, sous la houlette

5. R. Barthes, «Nautilus et Bateau ivre», Mythologies, op. cit., p. 80.

6. Fr. Jameson, Le postmodernisme ou la logique culturelle du capitalisme tardif [1992], ENSBA, 2007; Penser avec la science-fiction [2005], Max Milo, 2007-2008. 
de Bernard Blanc 7 , dans la collection «Ici et maintenant» en explicite opposition à celle qui domine le paysage éditorial du genre depuis sa création en 1969, "Ailleurs et demain » chez Laffont. C'est chez Kesselring que Jean-Pierre Andrevon, par exemple, publie en 1979 ce qu'il définit lui-même en sous-titre et en préface comme un "Retour à la terre 4" qui aurait changé de titre et d'éditeur entre-temps. Avenirs en dérive, cinquième et dernier collectif de cette aventure éditoriale (après des titres comme Ciel lourd béton froid ou Planète socialiste), offre un point de vue particulièrement intéressant sur le débat qui anima le milieu de la science-fiction française sur la critique de l'idéologie véhiculée par les artefacts et scénarios du genre. Il montre surtout à quel point ce débat fut interne, voire autodestructeur, ce qu'une trop simple opposition de titres de collections a tendance à occulter.

En 1977, Ciel lourd béton froid ouvre la série des collectifs sur un virulent plaidoyer :

Français jusqu'aux bouts des ongles. La SF d'ici ne sera pas une succursale de l'OTAN. [...] Autour de vous, le ciel est lourd et le béton froid. Faut-il vous mentir et vous raconter des histoires d'amour sur fond de musique douce, en technicolor publicitaire? Faut-il vous dire que la race humaine est splendide et ses dirigeants magnifiques? Faut-il vous montrer des fables où les extra-terrestres sont laids et dangereux? La SF a longtemps joué ce rôle d'usine à rêves, avec ses fantasmes réactionnaires et ses putasseries d'amuseuse publique. Une poignée d'écrivains français a décidé de renverser la vapeur [...]. S'ils faisaient dans le grand spectacle sanguinolent du génocide extra-terrestre, dans le film catastrophe plein de sauveteurs musclés [...] alors là, ils se rempliraient les poches. [...] Aujourd'hui, la SF casse les mythes et incendie les fusées. Aujourd'hui, elle parle des flics et de l'armée et explique qu'ils sont du mauvais côté, du côté des exploiteurs et des tyrans. Elle parle de la pollution et nomme les responsables : Rhône-Poulenc, EDF. et Péchiney. ATTACHEZ VOS CEINTURES! LA SF VA ATTERRIR ET ÇA VA SAIGNER ${ }^{8}$ !

Mettons en réserve pour l'instant l'allusion aux rapports culturels francoaméricains qui justifie qu'un repli national accompagne le discours militant : la fusée incendiée et son atterrissage fracassant dans le réel montre à quel point sont ici mises en coïncidence les "putasseries » mercantiles, l'hégémonie américaine et l'imagerie de la conquête spatiale. Les deux réseaux discursifs retiennent davantage l'attention : on y voit en effet ce qui organise le projet littéraire. Aux verbes mentir, raconter, montrer, jouer, amuser s'opposent casser, incendier, parler, expliquer, nommer. Dans cette opposition, c'est bien plus qu'une géopolitique culturelle (France vs USA), ou qu'une réorientation thématique (la terre $v s$ l'espace) qui s'exprime; c'est la parole contre le récit,

7. Auteur remarqué de l'iconoclaste Pourquoi j'ai tué Jules Verne, Stock, 1978.

8. Ciel lourd béton froid, collectif $\mathrm{n}^{\circ} \mathrm{I}$, Kesselring, I977; p. IO-I3, je souligne. 
la nomination contre le jeu, l'explication contre le mensonge. Il s'agira bien de "casser du mythe", et le "mythe" dont il s'agit englobe non seulement le silencieux récit naturalisant que traquait Barthes dans tel reportage de Paris Match ou telle photo de cuisine de Elle, mais aussi la forme narrative ellemême frappée ici d'indignité (mentir) - épisode ponctuel dans la longue histoire des anathèmes lancés contre la fiction romanesque, jeté ici contre son sous-genre spécialisé dans l'anticipation et le voyage spatial. Casser du mythe, ce sera donc casser les histoires (logos vs muthos), et en l'occurrence casser du futur (retour au présent). Dans les nouvelles, l'extrapolation minimale d'un futur proche, l'abondance de dialogues et de descriptions didactiques sont parfaitement congruentes à ce projet. Des banlieues HLM ou pavillonnaires, des centrales nucléaires ou des sites industriels en sont le décor privilégié; l'intrigue se résume le plus souvent à faire la guerre contre des gendarmes, des robots, ou les deux, non sans une jouissance destructrice qui détrompera vite tout lecteur ayant confondu le refus des "génocides extra-terrestres» avec la non-violence du flower power. La nouvelle de Philippe Curval, «Les communes", est sans doute celle qui pousse le plus loin l'exploitation de ces éléments : vingt pages de dialogue didactique entre Gery et Broco (non initié) se concluent sur la fabrication express d'un «masturbateur» en plastique moulé directement (par une femme blonde) sur son pénis en érection (une page); revêtu de cette armure qui garantira son excitation permanente, et équipé d'un «mange-béton», Broco s'en va détruire des villas en éjaculant (une page).

On aura beau jeu de dénicher, ici ou là, dans les quatre collectifs d' "Ici et maintenant", des nouvelles échappant à la caricature. Dans l'avant-propos d'Avenirs en dérive (dont le titre renoue avec une thématique anticipatrice, mais dont la dernière section s'intitule «Ici et maintenant»), c'est Jean-Pierre Andrevon lui-même qui se charge d'effectuer une sévère prise de recul, deux ans après le début de la série. Le projet d'une «SF en prise directe sur le réel, une SF qui fasse l'idéologie au lieu de seulement la refléter, une SF qui n'ai $[s i c]$ pas peur de s'étrangler en prononçant le mot politique», ce projet n'a pas convaincu. Les anthologies Retour à la terre se sont mal vendues, les lecteurs ont vilipendé ce courant littéraire dans les organes du fandom et la controverse a enflé : «l'arrivée massive de jeunes acteurs qui, à force d'être "en prise directe sur le réel", ont peut-être un peu trop perdu le sens de l'imaginaire, a provoqué une contre-réaction ${ }^{9}$ ". Le détail de cette "contre-réaction" vaudrait une analyse approfondie, qui montrerait la complexité des lignes de partage entre tenants et opposants d'une SF militante ${ }^{\text {Io }}$. Le nœud du

9. En gras dans le texte.

Io. Ph. Curval est aujourd'hui défendu et édité par G. Klein... 
problème n'est toutefois pas politique, et c'est là qu'il nous intéresse : «Alors oui, certains parmi nous ne sont pas exagérément optimistes... Cela veut-il dire qu'il faut faire de la mauvaise littérature avec nos bons sentiments, qu'il faut continuer à se lamenter noir sur blanc, à jeter nos anathèmes dans des anthologies-tracts? Sûrement pas non plus.» La question est bien celle d'une bonne ou mauvaise littérature, notamment dans son "sens de l'imaginaire», et de ce que doit devenir le "cassage de mythes" dans la science-fiction ${ }^{\text {II }}$.

L'aventure de la "nouvelle science-fiction française politique» me semble permettre, dans l'échec même de son ambition, de comprendre la portée possible (mais nullement, on le voit, certaine) de l'anticipation romanesque en termes de discours démystifiant. L'écrivain Roland C. Wagner, en réponse à un article de Jean-Christophe Rufin stigmatisant la «tentative peu concluante de créer une science-fiction strictement politique», a proposé récemment un double distinguo intéressant. Premièrement, souligne-t-il, la NSFFP n'a pas subsumé à elle seule tous les romans à portée politisante de la science-fiction ; elle en est une sorte d'avant-garde caractérisée par une instrumentalisation du genre, marquée par une opposition structurante à ce qui se publiait notamment au Fleuve Noir, et condamnée à l'impasse après l'arrivée de la gauche au pouvoir en 198I. Deuxièmement, et il y insiste, Wagner fait un parallèle entre cette avant-garde et une autre, celle du groupe Limite publié chez Denoël dans les années $198 \mathrm{O}^{\mathrm{I2}}$ :

M. Rufin accrédite l'idée malheureusement assez répandue qu'elle a exercé une influence prépondérante sur le genre.

C'est faux. L'influence de la NSFFP se dilue en effet très vite dans la première moitié des années 1980 ; c'est alors que commence le phénomène de rejet de cette période et de la SF militante : on se met notamment à accuser les auteurs " politiques " d'avoir fait fuir les lecteurs - une accusation dont il est symptomatique qu'elle fut reprise à la fin des années i980 à l'encontre de ceux que Bruno Lecigne avait baptisés les "néo-formalistes ", qui avaient alors pris le relais de la NSFFP en substituant « littérature " à " politique ".

Seulement, malgré tout le bruit que ses aficionados ont pu faire, le néoformalisme n'était pas plus que la NSFFP un mouvement véritablement dominant. Je verrais plutôt ces deux tendances comme des avant-gardes engagées dans une impasse. La NSFFP s'est retrouvée face au mur en 1981, le néo-formalisme, qui s'est développé à l'époque de son agonie, n’a pas survécu aux années $1980^{13}$.

II. J.-P. Andrevon, préface de Avenirs en dérive, collectif n ${ }^{\circ}$ 5, Kesselring, I979, p II-I3.

I2. Fondé par E. Jouanne, le groupe Limite comprend aussi L. Evrard, Fr. Serva, J. Barbéri, J.-P. Vernay, A. Volodine, Fr. Berthelot. Son but est de dynamiter les codes formels et esthétiques de la SF. Il publie Malgré le monde, anthologie collective, en 1987, chez Denoël (coll. «Présence du futur»).

I3. R. C. Wagner, «Science-fiction", Le Monde diplomatique, novembre 2004, p. 2; en réponse à l'article de J.-Chr. Rufin, "Réalité en quête de fiction ", Le Monde diplomatique, septembre 2004, p. 28. 
Cet épisode d'histoire littéraire symptomatique du «devenir roman» des démystifications semble ainsi proposer une double leçon : l'impasse de la NSFFP ne se comprend qu'en contexte, et relève peut-être autant, voire plus, d'un problème de réglage littéraire que d'une conjoncture politique. Le destin malheureux du groupe Limite (mais pas de tous ses membres : voyez Antoine Volodine) le suggère : la science-fiction ne supporte peut-être pas très bien l'expérimentation avant-gardiste (littéraire ou politique), et impose à l'écrivain un code romanesque passablement contraignant, qui sache ne pas «perdre le sens de l'imaginaire» (Jean-Pierre Andrevon) - ce qui n'est pas une mince affaire en général et dans la dénonciation des artefacts «mythologiques» en particulier. Gageons avec Roland C. Wagner que lorsqu'elle est travaillée par la SF avec le bon réglage, c'est-à-dire dans une articulation assez précise de la contrainte narratologique spécifique au genre et de la conscience des mythes contemporains, l'anticipation romanesque affirme et a affirmé la puissante efficacité d'une pensée spécifiquement littéraire : démystificatrice parce que romanesque, effectuant le montage narratif des «mythologies» du temps présent en même temps qu'elle offre, métatextuellement, la possibilité de les démonter.

Car il s'agit bien ici d'interroger précisément, sous le titre du «devenirroman des Mythologies", une fortune possible de cette forme spécifique de la démystification barthésienne, qui évite l'écueil de la "dénonciation pieuse» et trouve une voie textuelle capable de ménager, dans l'épaisseur romanesque, le temps d'un bonheur du mythe, d'une adhésion au mythe. De deux manières radicalement opposées, les science-fictions «de droite» et "de gauche» des années 1950 à 1970 ont fait l'économie de ce temps d'adhésion productive : l'une en transposant mutiquement les scripts et artefacts de la société de consommation triomphante dans un futur qui ne les interroge pas et les reconduit mécaniquement, pire encore : en fait les repères invisibles d'une activité imaginante déportée vers des audaces techno-scientifiques idéologiquement inoffensives; l'autre en rabattant l'extrapolation futuriste sur un démontage en règle du temps présent et en enrôlant les procédés de l'anticipation dans l'ensemble d'un discours globalement militant. Ici le bonheur du mythe est d'emblée stigmatisé comme aliénant; mais là, il est entretenu comme condition non négociable de possibilité d'un futur. Dénonciation pieuse d'un côté, aliénation douce de l'autre : le roman d'anticipation, qui s'énonce «depuis» le réel, ne peut sans doute rencontrer le puissant effet démystificateur des Mythologies qu'en se frayant une voie entre ces deux écueils. Certaines de ses réalisations montrent qu'il en a la capacité, tout à fait unique en son genre.

L'anticipation romanesque peut en effet proposer à l'imaginaire du lecteur toute la gamme des devenirs possibles de ses mythologies personnelles; et 
si elle réussit à encoder ces devenirs dans la narration suggestive, abductive, d'une énigme des temps futurs que l'imagination du lecteur doit silencieusement, au fil de la lecture des péripéties, élucider touche après touche, alors le temps de la narration, le temps de l'élucidation et le temps de la lecture peuvent se cumuler en lente - et jouissive - temporalité critique. Un bon synopsis en composera in fine le dénouement indissociablement diégétique, narratif et critique, pour une démystification activée au cœur même du bonheur des mythes, de leur imaginaire perduration future et du récit romanesque de cette perduration. Un cantique pour Leibowitz (A Canticle for Leibowitz, Walter M. Miller, I96I) met à contribution, par exemple, toute l'efficacité du roman de quête pour faire adhérer le lecteur à l'étrange obsession d'un «moine» d'une époque postcataclysmique. Trois chapitres diégétiquement distants de 600 ans chacun narrent, en trois touches historiques, le destin d'un homme fasciné par un texte mystérieux, la manière dont il entreprend un voyage destiné à le déchiffrer, puis les aléas de ce voyage et de ce déchiffrement. Immergé dans un néo-Moyen Age qu'aucun discours ne vient expliquer, le lecteur accomplit mentalement les raccords cognitifs lui permettant de comprendre le destin du personnage et du document. De façon caractéristique, les béances chronologiques de la diégèse, qui ajoutent en apparence au mystère historique, permettent en fait de multiplier les indices dont a besoin le lecteur. La progression dans le futur repose sur une production continue d'hypothèses destinées à relier les différentes époques du roman, une élucidation progressive des indices qui le permettent, le tout intimement imbriqué à l'histoire de personnages psychologiquement très approfondis. Durant tout le temps du roman, une immersion dans les coutumes, règles, lois et anecdotes de cet autre monde se produit; l'identification aux personnages achève l'adhésion. La quête du sens de ce texte indéchiffrable devient donc aussi celle du lecteur, dans un procédé, classique et ici très soigneusement mis en place, de roman à énigme. Les I200 années du temps de l'histoire reflètent en elles-mêmes l'écart prodigieux que doit mentalement accepter le lecteur, et qui aiguisent son intérêt.

Or les découvertes de chaque chapitre offrent toutes un retournement spectaculaire de tous ces procédés d'adhésion, et se chargent d'un message qui en démystifie cruellement les conclusions. Le texte mystérieux se révèle être un document issu de l'époque précataclysmique - la nôtre, donc - et porteur de tous les éléments technologiques qui permettront à ses lecteurs de reconstruire la technoscience qui a causé le cataclysme... cataclysme dont la société néomédiévale du roman avait précisément pour tâche d'empêcher toute résurgence. Le pessimisme final du roman (un paysage de désolation) s'exprime d'une manière étroitement imbriquée à sa structure romanesque, 
qui exploite les codes de la "boucle temporelle»(time loop) très appréciée dans les histoires de voyage dans le temps. Ici l'usage en est destiné à une leçon sur les dangers de la science sans conscience; le message est ordinaire, la manière de le délivrer, beaucoup moins. Elle passe par une observation fascinée, puis progressivement alarmée, et enfin, littéralement, catastrophée, de la croissance, de l'apogée et du déclin de cette société fictive décalquant, sans le savoir, le processus d'une première apocalypse! Le lecteur est chargé, lui, de ce savoir mi-interne, mi-externe à la fiction : l'anticipation romanesque l'y autorise, faisant du temps présent l'origine absente d'une histoire que la diégèse ne peut pas remettre à jour - et dans laquelle le lecteur, lui, ne peut pas ne pas faire jour. Le tour est joué, et la boucle est bouclée.

Ce que le lent cheminement de ces moines imaginaires accomplit, une «mise en tableau » bien faite peut aussi y parvenir, dans le vaste corpus de ce qu'il est convenu d'appeler les dystopies. En effet, le figement d'un ordinateur, d'un hypermarché ou d'une émeute urbaine en "image évidente» d'un tableau de société acquiert, avec la projection de ce tableau dans le futur, un sens nouveau. Les mythologies contemporaines y connaissent un parfois subtil traitement de constitution/destitution : certaines y sont nominées, sans qu'aucun récit ne vienne justifier cette élection dans le composé délétère d'une description sinistre de monde futur repoussant. Si le lecteur cherche donc à retracer mentalement l'historique imaginaire de cette perduration (et c'est le contrat générique indiscuté du mode science-fictionnel), il ne peut que dessiner, en même temps que la figure d'un mythe sélectionné dans la foule de ses habitus, que la courbe d'une dévalorisation révélatrice : dans le tableau dystopique, la seule mention de cette mythologie particulière a valeur de jugement sans appel, quand bien même - et surtout si - aucun discours militant ne vient appuyer la condamnation sociétale. Le tableau, dans ce cas, vient faire sens en tant que tel, et «l'effet de réel» ainsi créé monte et démonte en un seul geste narratologique le contenu idéologique d'un item. Autrement dit, ce cas de figure parvient à l'effet «mythologique» par un biais exactement inverse de l'aménagement temporel qu'on soulignait plus haut. John Brunner, dans sa "tétralogie noire» (quatre romans brossant la fresque pessimiste d'un futur proche passablement repoussant), adopte pour quasi seul procédé descriptif le montage d'extraits (slogans, inserts, documents fictifs...) et instantanés ou esquisses de faits divers, portraits d'anonymes ou paysages urbains non développés. Le style "coup-de-poing" qu'en acquiert son roman est doublement avantageux : d'une part il élude les justifications et motivations diverses (narrative, mais aussi économique, sociale...) de ses choix, d'autre part il met régulièrement le lecteur face à des aperçus globalement connotés négativement mais laissés à sa libre interprétation 
historique, archéologique (si l'on prend le point de départ fictionnel, situé dans le futur) - ou prospective, si l'on admet que le présent reste le seul point de départ effectif. Là est évidemment la clé de cette efficacité "mythologique», au plein sens barthésien du terme (jouissance du mythe et déconstruction de celui-ci sont concomitants dans l'opération de prise de conscience "sémioclastique») : les coups de projecteur sur des composants du futur dystopique déclenchent à la fois une invitation à leur archéologie imaginaire et un exercice de futurologie qui est, on le voit bien, la seule armature logique de l'illusion archéologique. De façon redoutablement antagonique, les deux opérations temporelles se croisent dans l'esprit du lecteur, accomplissant soit la fermeture d'un futur toujours-déjà rapporté au présent dont il est la projection, soit l'ouverture d'un "passé» de la fiction perpétuellement rajeuni par sa redéfinition en présent de la lecture, soit... les deux.

C'est pourquoi ce procès descriptif dystopique, apparemment contraire à la progressive temporalité critique du temps barthésien, représente un «devenirroman » si efficace des Mythologies. Le principe anticipatif est comme la « réserve de temps critique» livrée avec chaque item de la sélection. Il n'est d'ailleurs pas nécessaire que cette réserve soit thématisée par une projection extrême dans un futur très lointain. De façon tout à fait caractéristique, aussi bien les pamphlets militants d' «Ici et maintenant» que les œuvres d'inspiration cyberpunk ou la tétralogie brunnérienne adoptent une science-fiction du futur proche (near future). Des romans comme Neuromancer, Pattern Recognition (William Gibson), Temps blancs (Jean-Marc Ligny) ou Le travail du furet (Jean-Pierre Andrevon) adossent leur portée critique à une projection futuriste qui peut se contenter, et même fleurit sans doute mieux, dans son degré zéro qu' est "l'écart " temporel, aussi peu datable qu'indubitable. L'activité de projection futuriste des spectateurs d'un film comme Blade Runner (adapté de Philip K. Dick) est autant sollicitée par l'exotisme des voitures volantes que par la présence de boutiques de plats chinois à emporter, incongrûment pérennes. L'anticipation, même minime, du fait de son irréductible fonctionnement temporel, propose le même effet de loupe sur des éléments de notre présent (publicités, objets manufacturés, comportements, désirs) que les contemplations ravies, puis ressaisies, des fifties barthésiennes. Ni dénonciation pieuse ni aliénation douce, la dystopie mise sur l'effet perturbateur d'un élément du présent transporté tel quel, ou quasi, dans un futur globalement négatif.

La manipulation temporelle exigée par l'anticipation représente ainsi un procédé critique très original, non pas parce qu'elle transpose dans un futur fictif (forcément) des diatribes contemporaines du lecteur (à moins que l'objet à critiquer, et son devenir-mythe, soit précisément la diatribe gauchiste militante), mais parce qu'au contraire elle ne dit pas grand-chose de ce qui perdure 
dans le monde futur de la fiction, ou de l'histoire de cette perduration. Si l'on n'en était pas encore tout à fait convaincu, on pourra effectuer un dernier relevé dans la petite fabrique de ruines dont la science-fiction est une des plus actives usines littéraires. Ces ruines peuvent être de nature différente, elles n'en sont pas moins caractérisées par le même fonctionnement : un item mystérieux de la fiction futuriste finit par être compris, soit d'une façon brièvement esquissée, soit d'une façon plus approfondie, comme le reste en ruines d'un item d'une époque lointainement passée (pour l'univers fictionnel), proche de son présent (pour l'univers du lecteur). Ruines du langage, dans les hurles et les grognes d'un Pierre Bordage (Les Fables de l'Humpur) ou l'onomastique du roman postcataclysmique ("Litale» d'Élisabeth Vonarburg dans les Chroniques du Pays des Mères, Niourk de Stefan Wul); ruines du bâti, que ce soit le bâti classique des monuments et constructions urbaines ou le bâti plus diffus des routes, des infrastructures et des bien nommés ouvrages d'art; ruines du fait social et symbolique, dans l'herméneutique assidue qu'un groupe de chiens du lointain futur effectue sur un corpus de fables (City, de Clifford D. Simak). Ruines du corps humain, enfin, voire de sa structure génétique profonde, dans l'abondante littérature de la mutation et de la posthumanité. Un procédé si fréquent et si congruent au genre du roman à énigme qu'on en oublie parfois que, dans sa catégorie anticipatrice, le roman à énigme appliqué à l'investigation des ruines est une approche originale du présent, chargé de toute l'efficience paradoxale que la littérature reconnaît aux ruines, de Pétrarque à Chateaubriand en passant par Du Bellay, Diderot ou Keats : dans le spectacle de sa destruction, la ruine offre au voyageur la double face d'une splendeur passée et du moignon qu'il en demeure, ou si l'on préfère du reste pitoyable de la splendeur qui indique en même temps que cette splendeur est passée mais qu'elle fut assez puissante pour en laisser des traces. Comme dans $U n$ cantique pour Leibowitz de Walter Miller, on invite le lecteur au comblement d'une béance historique, selon des modes longuement (si le roman est une "archéologie-fiction") ou incidemment narrativisés. Dans certains cas, opportunément disponibles à l'industrie cinématographique qui en a bien retenu l'avantage en termes de réalisation, un simple aperçu de la ruine peut déployer le paysage allusif entier d'une catastrophe : piscine vide, immeuble de grande taille dont les vitres sont brisées, bitume d'une voie rapide envahi par les herbes. James Graham Ballard est un orfevvre en la matière, prisant particulièrement l'expressivité du végétal non maitrisé, qui dit en un seul geste, là encore, le soubassement d'une civilisation et le descriptif de sa ruine. L'abandon (des villes, des bâtiments, de la civilisation) n'est toutefois pas le seul synopsis possible, et le roman peut alors embrasser le projet d'une enquête, adopter le point de vue d'un personnage du futur, et placer le lecteur dans une position très 
comparable à celle de l'ironie tragique. La fiction anticipatrice autorise en effet, dans ce cadre, tous les jeux de dissymétrie cognitive au sein desquels trouvera à s'exercer la lucidité du temps présent, dans tous les sens du terme : lucidité du lecteur sur son propre présent, développant peu à peu une futurologie orientée par les énigmes du roman; lucidité des personnages de la fiction sur leur passé, qui se trouve être le présent du lecteur - ainsi, parfois complètement dépassé, parfois tragiquement informé.

En tant que telle, la fiction anticipatrice offre un spectaculaire pendant à l'art ancien des Vanités. La mort en effet n'est pas le seul scénario possible du crâne retrouvé sur une lointaine planète (ou dans New York - Niourk - en ruines); des civilisations extraterrestres disparues pour mieux revenir aux boucles temporelles des voyages dans le temps, voire aux brassages des uchronies, les choses restent largement ouvertes dans le domaine des littératures de l'imaginaire en général et dans la science-fiction en particulier, où le "sarcasme» démystifiant peut aisément trouver à nourrir sa chasse aux mythes sans virer à l'aigreur. On lira pour s'en persuader l'extraordinaire paysage du «Golden Gate à Machu Picchu» brossé par Dan Simmons dans sa passablement loufoque réécriture d'Homère et de Virgile - Ilium étant toutefois aussi, entre autres, une broderie sur Ada ou l'ardeur et les Sonnets de Shakespeare ${ }^{\mathrm{it}}$. Transporté dans les ruines incas pour une raison que le narrateur se garde bien s'expliquer (c'est un mystère absolument marginal dans l'énigme complexe du roman), le Golden Gate en acquiert un statut de ruine exemplaire bien sûr, mais jette également, par ricochet (et notamment parce qu'on se demande quand même ce qu'il fait là et comment on l'y a amené), une lumière nouvelle sur la cité inca, la redynamise pour ainsi dire, et l'embarque dans une événementialité imaginaire future. On ne saurait mieux à la fois moquer, chérir et, au sens propre, déplacer les mythologies du temps présent que sont, chacun à leur manière, et le Machu Picchu (engouement savant, puis populaire, pour les "antiquités du Nouveau Monde», délires ufologiques, tourisme de masse) et le Golden Gate (ingénierie des ponts, «sept merveilles du monde moderne», Grand Ouest américain).

Ces ruines drôlatiquement, mélancoliquement ou âcrement démystificatrices ne sauraient ainsi dicter la conclusion que le roman anticipateur ne met son potentiel critique qu'au service du désenchantement. Si la science-fiction mérite qu'un panorama du roman contemporain s'y attache, c'est peut-être, pour finir, autant pour ce quautorise le potentiel critique de sa projection futuriste, que pour la jubilation d'un esprit critique éternellement continué

I4. D. Simmons, Ilium [2003], trad. J.-D. Brèque, coll. "Ailleurs et demain", Laffont, 2004. Les passages du Machu Picchu sont aux chapitres I6 et I9. 
qu'elle met parfois - dans ses meilleures œuvres - en scène : survie de la jouissance des mythes au-delà de leur démystification, survie de la lucidité critique renourrie d'imaginaire, et mutation du «sarcasme» barthésien en éclat de rire androïde, fût-il improbablement post-humain. Évoquons une dernière fois Ilium, dont le message semble dire : quitte à mythifier un supposé Shakespeare gay dans les Sonnets, autant en faire une histoire d'amour. Entre Mahnmut et Orphu, deux moravecs en orbite de Jupiter, êtres posthumains mi-robots, mi-ordinateurs, mi-mémoire informatique? Passionnés d'herméneutique littéraire et se livrant au fil des millénaires à une exégèse croisée de l'amour chez Marcel Proust et "Will»? Violemment rapprochés par une explosion quantique et découvrant à cette occasion à quel point ils s'aiment ${ }^{15}$ - qu'importe? C'est toujours de l'amour... et de la littérature!

I5. Les passages marquant le plus nettement la progression de leur "amitié» sont aux chapitres 12 et I4; ensuite, ils fusionnent en raison des avaries infligées à Orphu... 